\title{
STIFFNESS AND DAMPING CHARACTERISTICS OF MR FLUID-BASED SANDWICH BEAMS: EXPERIMENTAL STUDY
}

\author{
Mateusz Romaszko, Bogdan SAPIŃSki \\ AGH University of Science and Technology, Department of Process Control, Cracow, Poland \\ e-mail:matek28t@poczta.fm; deep@agh.edu.pl
}

\begin{abstract}
The study investigates the behaviour of three-layered cantilever sandwich beams filled with magnetorheological fluids (MRFs) differing in the iron particle content by volume. Outer layers are made of aluminium, the space between them is sealed with silicone rubber. Two types of beams are considered: fully filled beams and partially filled beams, subjected to the magnetic field. The aim of the study is to determine stiffness and damping characteristics in relation to the magnetic field strength and the actual location where the magnetic field acts upon the beam. For this purpose, measurements have been taken of the beam free vibration response for various magnetic field strength levels and for various positions of the electromagnet located along the beam axis. Basing on the developed measurement data processing algorithm, the influence of the vibration amplitude on the natural frequency and a dimensionless damping coefficient have been determined. Finally, the equivalent natural frequency and the dimensionless damping coefficient have been derived accordingly, and the stiffness and damping ratio have been determined in function of the magnetic field strength, the electromagnet position and the MRF iron particle content by volume.
\end{abstract}

Keywords: magnetorheological fluid, beam, magnetic field, vibration, stiffness, damping

\section{Introduction}

Beams, plates and shell elements are widely used as structural components in a variety of mechanical systems. Their operation, however, gives rise to some undesired phenomena, such as noise or vibrations, leading to damage due to material fatigue. In most systems, stiffness and damping characteristics of their structural components are associated with the type of the manufacturing material. Stiffness and damping characteristics mostly remain unchanged except when the elements have sustained some damage or when they were exposed to action of external factors. In order that stiffness and damping characteristics should be effectively controlled, their structural components are modified to integrate some smart materials, such as magnetorheological fluids (MRF), electrorheological fluids (ERF), piezoelectric elements or other. Yalcintas and Dai $(1999,2004)$ analyzed dynamic responses of a MRF adaptive structure and compared it with that of ERF containing structure, and established that MRF-based adaptive structure could yield natural frequencies two times higher than ERF-based adaptive structures. Sun et al. (2003) determined dynamic characteristics of MRF sandwich beams experimentally and recalling the energy approach. Yeh and Shih (2006) modified the theoretical model of a simply-supported MRF-based adaptive beam under an axial harmonic load, recalling DiTaranto (1965) sandwich beam theory for a symmetric three-layer beam. The incremental harmonic balance method was utilised to identify dynamic instability regions. The study investigated how the magnetic field and beam dimensions should affect the buckling load, natural frequency, loss factor and dynamic instability. Rajamohan et al. (2010c) derived the finite element approach and Ritz formulation for a sandwich beam with MRF treatment and showed their validity through tests done on cantilever sandwich beams. Rajamohan et al. (2011) formulated an optimal control strategy based 
on a linear quadratic regulator and a full dynamic observer to suppress vibration of a cantilever beam with full and partial MRF treatments under a limited magnetic field. All those studies investigated beams with uniform MRF layers subjected to a uniform magnetic field.

A few papers published recently demonstrated that non-uniform MRF treatment could be beneficial for vibration suppression under the transverse excitation. Lara-Prieto et al. (2010) conducted an experimental study of the dynamic response of a MRF sandwich cantilever beam under the action of a uniform and non-uniform magnetic field, showing that the natural frequency of the beam tends to decrease as the permanent magnets are moved towards the beam free end. Rajamohan et al. (2010a) formulated equations governing the behaviour of partially treated MRF sandwich beams in varied configurations and for different boundary conditions using the finite element approach and Ritz formulation. The results clearly suggest that the natural frequencies and the transverse displacement response of partially treated MRF beams are strongly influenced not only by the magnetic field strength but also by the location and length of the MRF pocket. Next, Rajamohan et al. (2010b) considered various configurations of sandwich beams partially treated with MRF, including a beam with a cluster of MRF pockets, a beam with arbitrarily located MRF pockets and with different end conditions. An optimisation problem was formulated by combining finite element analysis with optimisation algorithms based on sequential quadratic programming and a genetic algorithm to identify optimal locations of MRF treatment to achieve the maximal modal damping in the first five modes of flexural vibration. The results showed that the optimal location of the MRF treatment was strongly related to the beam end conditions and the vibration mode. Rajamohan and Ramamoorthy (2012) investigated the vibration behaviour of a multi-layer beam structure comprising an axially non-homogeneous MRF layer in terms of natural frequencies and the loss factor associated with particular modes for varied locations of MRF treatment, varied boundary conditions and subjected to magnetic fields of varied strength levels. It was established that natural frequencies and the loss factor of non-homogeneous MRF beams were strongly influenced not only by strength of the applied magnetic field but also by the actual location of the MRF treatment.

The previous research work of the authors was focused on homogeneous aluminium beams (Romaszko et al., 2015) and three layer beams containing MRF: two fully filled beams (Romaszko et al., 2011; Snamina et al., 2012a,b) and two partially filled beams (Snamina et al., 2012b). The present study summarises experimental investigation of four sandwich beams filled with MRFs (two fully and two partially filled beams). In the present study, the authors employ a newly designed electromagnet (Romaszko and Lacny, 2015). Besides, the end of each beam is clamped in a holder placed at the moving part of the electrodynamic shaker. A slight modification is introduced to investigate forced vibrations with vertical kinematic excitation of the holder (Romaszko et al., 2014). The same boundary conditions are preserved - the cantilever beam. The size of the electromagnet pole pieces cover $80 \mathrm{~mm}$ of beam length. Moreover, the gap between the poles is constant this time. The electromagnet is characterized by generation of a larger magnetic field strength and dynamically responding time implies faster reaction, and that is the key parameter for vibration control (Romaszko, 2013). Besides, in the current study the algorithm for determining natural frequency versus vibration amplitude of the beam and dimensionless damping coefficient versus the vibration amplitude is enriched with an additional parameter. It refers to the number of periods of damped vibrations. This parameter behaves similarly to a low-pass filter. The modified experimental set up enabled the authors to repeat and extend the experiments under new conditions.

The authors of the present study investigated experimentally the behaviour of four MRF treated sandwich beams (two fully treated and two partially treated). Two fully treated beams were filled with different types of MRF (characterized by various iron particle content by volume). The objective of the study was to determine the dynamic properties of those beams, particularly their stiffness - natural frequency and damping characteristics - dimensionless damping coefficient. 
The influence of the vibration amplitude on the natural frequency and a dimensionless damping coefficient were determined. Then the equivalent natural frequency and the dimensionless damping coefficient were derived accordingly and the stiffness and damping ratio were obtained as a function of the magnetic field strength (taking into account also the non-homogeneous magnetic field), the electromagnet position and the MRF iron particle content by volume.

The work is organised as follows. Section 2 outlines the structure and fabrication technique of beams. Section 3 summarises the experimental set-up and the scenario of experiments (free vibration response measurements). Section 4 presents the experimental data processing algorithm. Section 5 discusses stiffness and damping characteristics of the beams in terms of magnetic field strength levels, electromagnet position and MRF iron particle content by volume. Final conclusions are given in Section 6.

\section{Description of the beams}

\subsection{Structure}

The structural design and dimensions of the beam are shown in Fig. 1. Two outer layers made of aluminium are $400 \mathrm{~mm}$ long, $30 \mathrm{~mm}$ wide and $2 \mathrm{~mm}$ high. The space between the layers is sealed with silicone rubber ( $2 \mathrm{~mm}$ in height, $1.5 \mathrm{~mm}$ in width). The inside of Beam 1 and Beam 2 is fully filled with a MRF manufactured by Lord Corporation. Beam 1 contains 122-2ED fluid (MRF1) while Beam 2 with 132DG fluid (MRF2). MRF1 and MRF2 differ in the iron particle content by volume (MRF1 contains $22 \%, \mathrm{MRF} 2-32 \%$ ). An increase in the particle content in MRF gives rise to an increase in viscosity, density, and changes magnetisation properties and the yield point. Weight of Beam 1 and Beam 2 minus the fluid is $146 \mathrm{~g}$. When filled, Beam 1 weights $196 \mathrm{~g}$ and Beam $2-211 \mathrm{~g}$. In the case of partially filled Beam 3 and Beam 4, the space between the aluminium layers is divided into five identical pockets, one of them filled with fluid MRF2 (pocket 1 in Beam 3 and pocket 3 in Beam 4). Weight of Beam 3 and Beam 4 minus the fluid is $146.5 \mathrm{~g}$ and $159 \mathrm{~g}$ when filled.

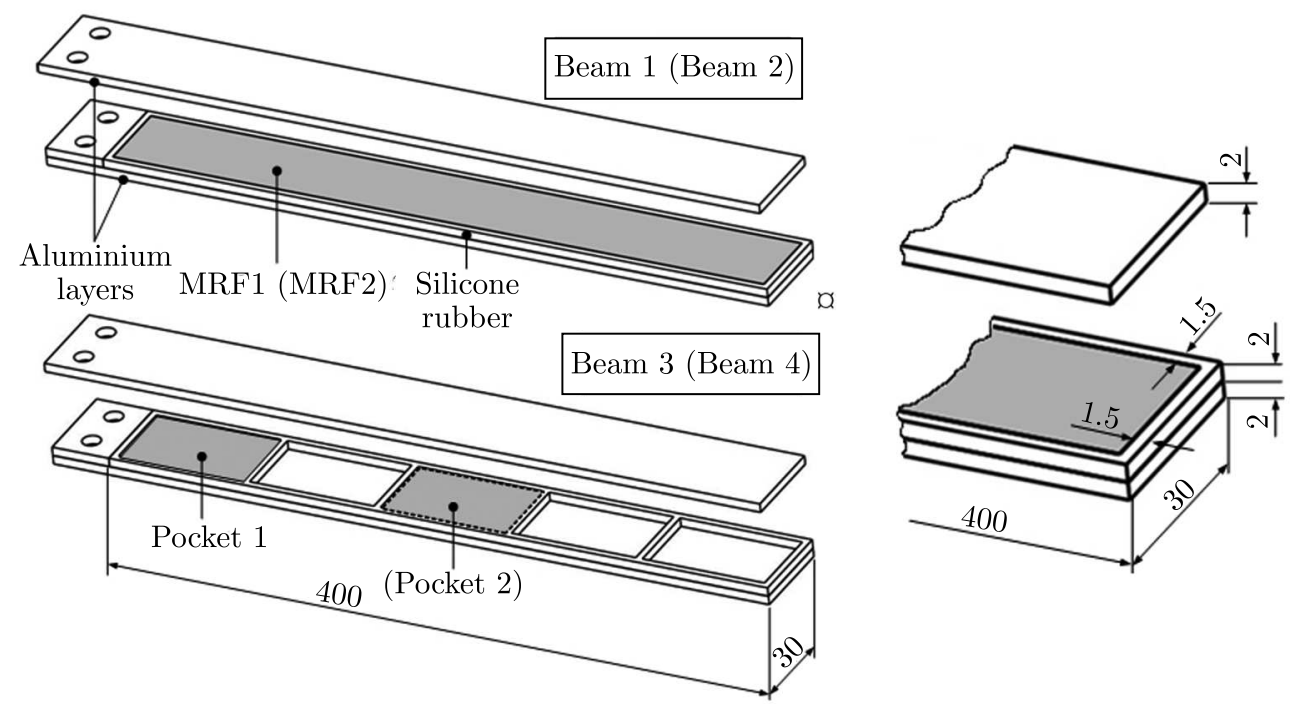

Fig. 1. Structure of the beams

\subsection{Fabrication}

The beam fabrication involved four stages. At stage 1, the aluminium sheet $30 \mathrm{~mm}$ in width was used and two elements $426 \mathrm{~mm}$ in length were cut out. During stage 2, those elements were carefully cleaned and silicone rubber was attached to their edges. In the case of Beam 3 and 
Beam 4, four transverse stripes of silicone rubber were attached with an adhesive, thus forming five equal pockets. One of the edges of pocket 3 in Beam 4 had silicone sealing removed at two points, along a section $2 \mathrm{~mm}$ in length. That procedure was unnecessary in the case of Beam 3 (pocket 1). Afterwards, the second layer of aluminium was attached. Stage 3 consisted in Beam 1 and Beam 2 of filling with MRF1 and MRF2 respectively, using a syringe. A similar procedure was adopted when handling Beam 3, and its pocket 1 was filled with MRF2. In stage 4, an aluminium insert was provided between the outer layers from the fixture end in Beam 1, Beam 2 and Beam 3. In the case of Beam 4, this operation was performed during stage 3 whilst in stage 4, pocket 3 was filled with MRF2, via one of the two opening. The other opening was used to ensure air removal from thus filled pocket. Pocket 3 having been filled, the openings were sealed with silicone rubber.

\section{Measurements of the free vibration response}

A schematic diagram of the experimental set-up is shown in Fig. 2. One end of the investigated beam is clamped in a holder located in the moving part of the electrodynamic shaker (not used in this study). The suspension of the shaker armature and the upper platform with holder have sufficient stiffness, which does not affect beam free vibration. The magnetic filed is generated by the current flowing in the electromagnet. The location of the electromagnet $y_{m}$ is determined by the distance between the beam fastening point and the mid-point of the electromagnet core of length $e=80 \mathrm{~mm}$. The electromagnet is allowed to move freely along the beam length. The measurement and control system incorporates a PC computer, an I/O board RT-DAC4 (INTECO Ltd.), a DC power supply, an amplifier specially designed for this application and a laser sensor SENSOPART RLA-70S1-L8. The system is supported by MATLAB/Simulink software. The power amplifier and I/O board enable control of the magnetic field strength $H$. The laser sensor measures the displacement $z$ of the beam free end.

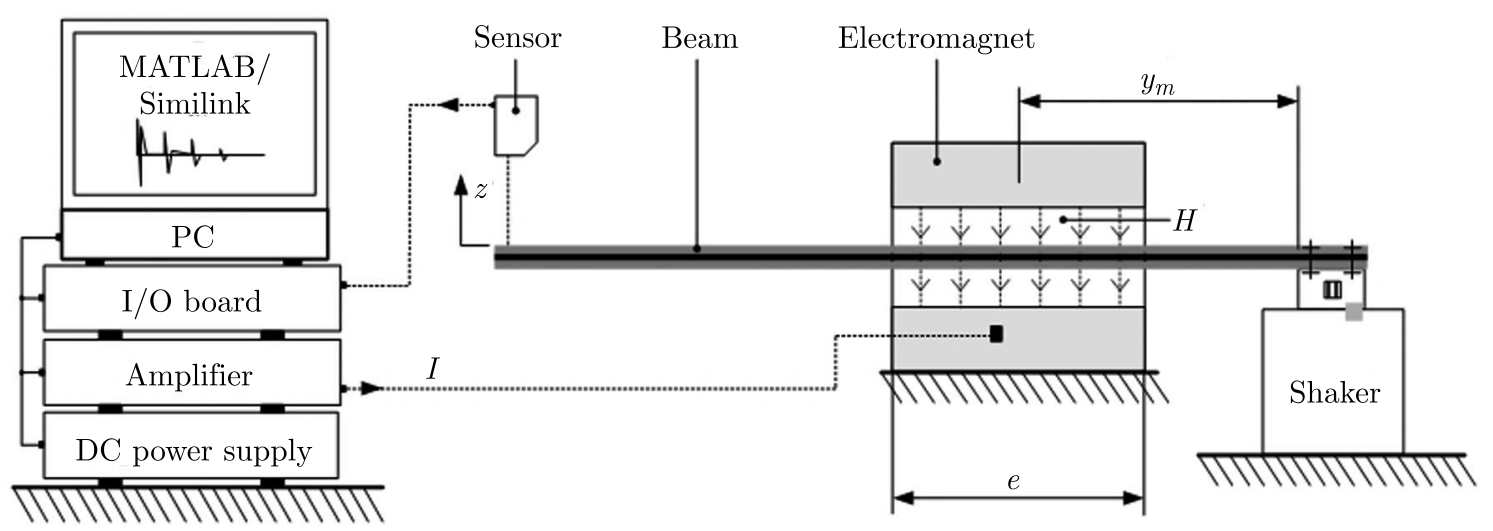

Fig. 2. Schematic diagram of the experimental set-up

Measurements of the beam free vibration response were taken by deflecting its free end from the equilibrium position and recording the displacement $z$ at s sampling frequency of $1 \mathrm{kHz}$. The measurements were performed for current levels in the electromagnet: $0,1,2.4,3,3.3,3.5 \mathrm{~A}$; the corresponding field strength levels $H$ were: $0,45,101,127,140,148 \mathrm{kA} / \mathrm{m}$ (Romaszko and Lacny, 2015). In the case of Beam 1 and Beam 2, measurements were carried out with the following position of the electromagnet $y_{m}$ : 40, 60, 80, 100, 120, $140 \mathrm{~mm}$ (Fig. 3a). For Beam 3 and Beam 4, the electromagnet position was assumed to be $y_{m}=40 \mathrm{~mm}$ and $y_{m}=200 \mathrm{~mm}$, respectively. These values of $y_{m}$ were related to which pocket was actually filled with MRF (see Fig. 3b). On account of the electromagnetic structure and action of a non-homogeneous magnetic 
field, the beam free vibrations were impossible to register when the electromagnet position was $y_{m}>140 \mathrm{~mm}$.

(a) Beam 1 or Beam 2

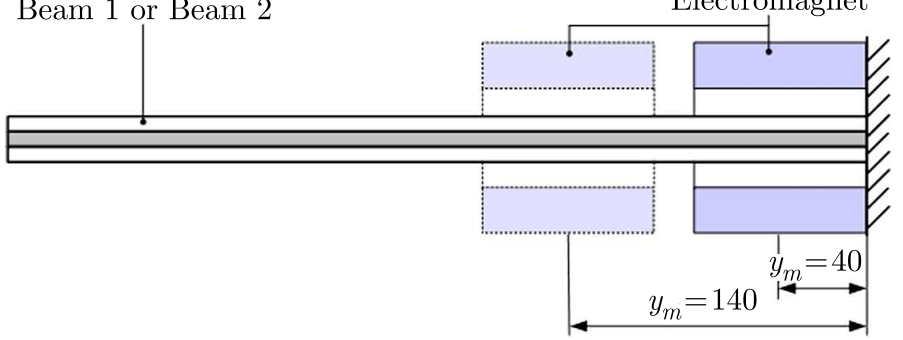

(b) Beam 1 or Beam 2

Electromagnet

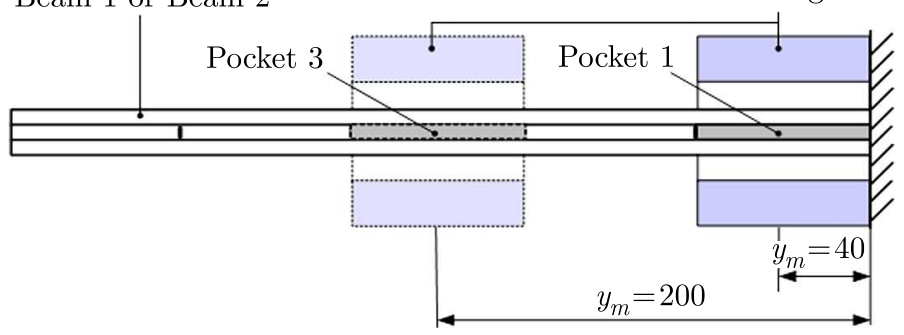

Fig. 3. Electromagnet position in the beams: (a) fully filled, (b) partially filled

(a)

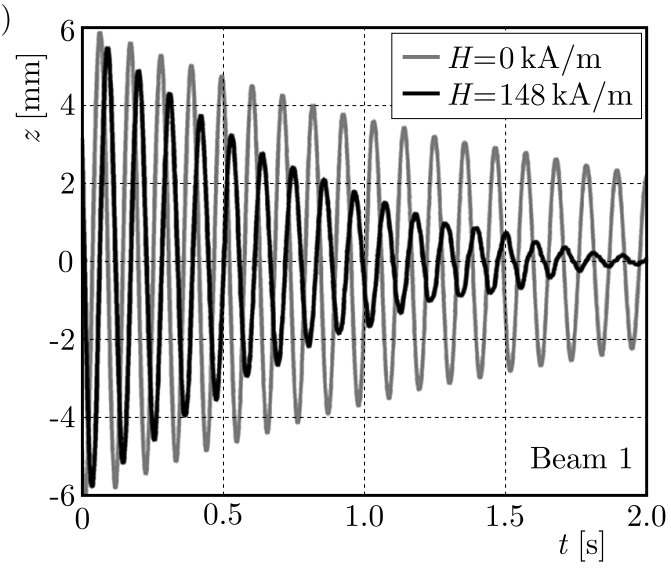

(c)

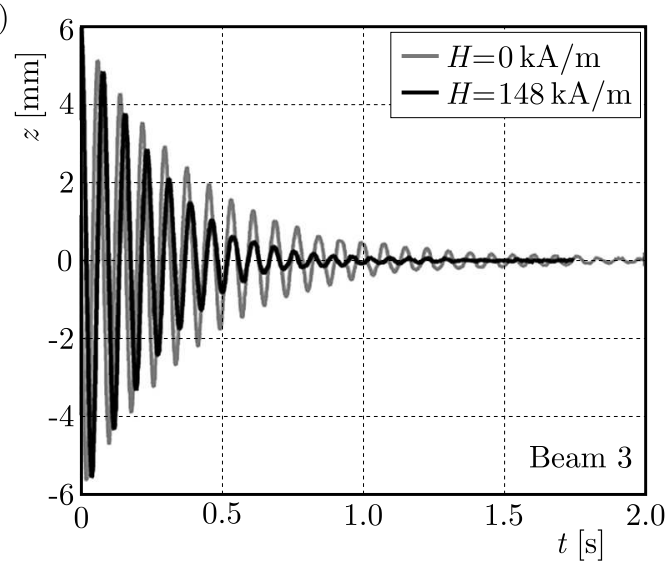

(b)

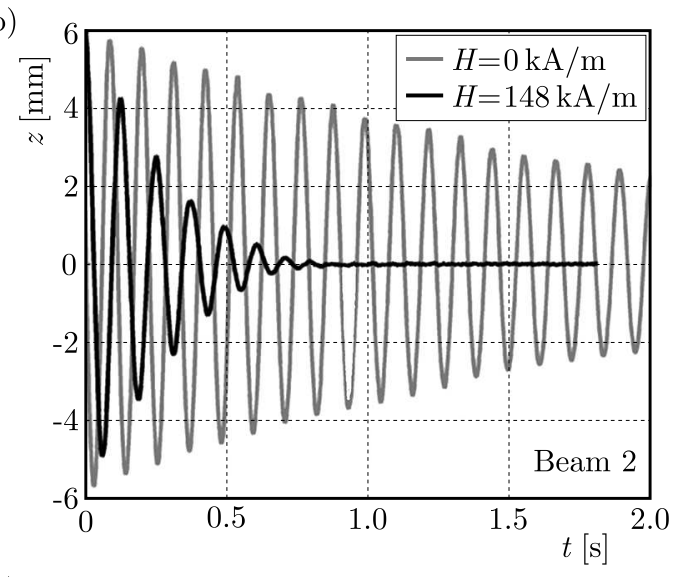

(d)

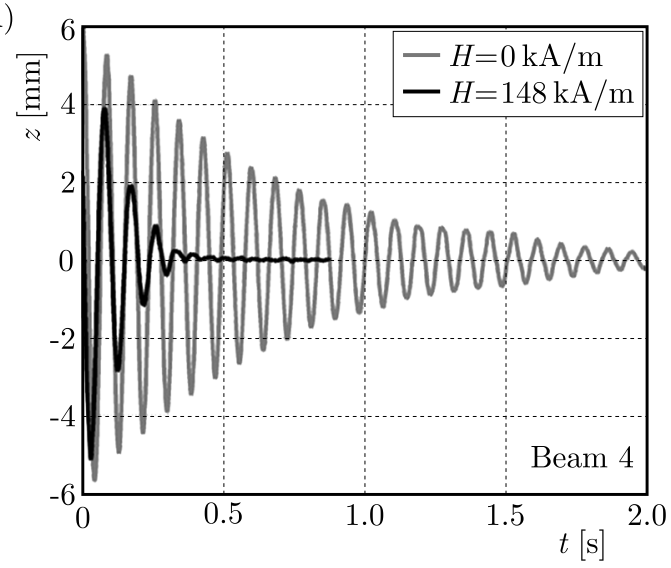

Fig. 4. Free vibration responses

Example free vibration responses of the free end of each beam are shown in Fig. 4, when $H=0 \mathrm{kA} / \mathrm{m}$ and $H=148 \mathrm{kA} / \mathrm{m}$. The plots for Beam 1 and Beam 2 are obtained for the electromagnet position $y_{m}=140 \mathrm{~mm}$. It appears that the decay time tends to decrease with 
an increased magnetic field strength level. Comparison of the decay time plots for Beam 1 and Beam 2 taken for the same magnetic field strength level clearly reveals that for Beam 2 the decay time is shorter, which can be attributed to the fact that MRF2 filling Beam 2 has a higher iron particles content than MRF1, which enhances damping. The analysis of measurement data suggests that for the maximal magnetic field strength $(H=148 \mathrm{kA} / \mathrm{m})$ the highest damping is registered for Beam 4. In order that interpretation of results should not be obscured by the presence of eddy currents (in aluminium layers) affecting the beam vibration parameters, further measurements were performed to investigate the behaviour of Beam 1 (not filled with MRF1) under the action of a magnetic field. The results showed that magnetic field strength had no effect on the beam vibration parameters.

\section{Processing of the experimental data}

The registered free vibration responses of the beams have been used to determine the natural frequency $f_{0}$ and the dimensionless damping coefficient $\zeta$ in function of the vibration amplitude $Z$. Thus obtained stiffness and damping characteristics were used to derive an equivalent natural frequency $f_{0 z}$ and an equivalent damping coefficient $\zeta_{z}$ in relation to the magnetic field strength level $H$ and the electromagnet position $y_{m}$.

In the first place, beam vibrations are damped by the MRF filling the beam. Damping properties of the MRF layer can be controlled through the action of the magnetic field. Several energy dispersion mechanisms are involved, including internal friction in aluminium layers and in sealing rubber, friction between the MRF layers and aluminium layers and friction between iron particles suspended in the carrier fluid. Further, energy dispersion is enhanced by external damping. Damping of the beam vibration is not strictly equivalent to viscous damping. It is suggested, therefore, that the measurement data should be processed in accordance with the algorithm, which yields the approximate natural frequency $f_{0}$ and the dimensionless damping coefficient $\zeta$ for the first mode in relation to the amplitude $Z$ of the registered displacement $z$. The data processing algorithm involves five steps.

Step 1 defines width of the window $k$ and the number $N$ of amplitudes $Z$ to be found. The amplitude $Z$ is found in the range of $0.5-6 \mathrm{~mm}$ and that enables determination of the number $N$ of amplitudes. The number $k$ corresponds to the number of periods of damped vibrations. Next, the signal $z$ is subjected to low-pass and high-pass filtering using the 1st order analogue filters. The critical frequency is chosen such that the frequency band from $2-80 \mathrm{~Hz}$ should remain.

In Step 2, the subsequent amplitudes $Z_{n}$ are found.

In $\overline{\text { Step } 3}$, the window is introduced with the width $k$, which is shifted along the time axis. The beginning of the window is set at the time instant when the amplitude occurs $Z_{n}$ $(n=1,2, \ldots, N)$. For each subsequent amplitude $Z_{n}$ that is thus located, the time instant $t_{n}$ when it occurs is recorded. An example of the free vibration response is shown in Fig. 5, revealing the movable window of the width $k$, the amplitudes that have been found $Z_{n}, Z_{n+k}$ and the time instants they occur.

The introduced quantity $\Delta_{n k}$ is used to determine the logarithmic damping decrement. It is assumed that in the interval between the time instants $t_{n}$ and $t_{n+k}$ when the respective amplitudes $Z_{n}$ and $Z_{n+k}$ are registered, the beam damping can be approximated by viscous damping. That is why the damping coefficient and natural frequency in the investigated interval are constant. The quantity $\Delta_{n k}$ is expressed by the formula

$$
\Delta_{n k}=2 \pi f_{0 n} \zeta_{n} k T_{n}
$$

where $\zeta_{n}$ and $f_{0 n}$ denote the dimensionless damping coefficient and frequency of undamped vibration (natural frequency). In the investigated interval, beginning at the instant $t_{n}$ and 
corresponding to the occurance of amplitude $Z_{n}$, the average period of damped vibrations $T_{n}$ is derived from the formula

$$
T_{n}=\frac{t_{n+k}-t_{n}}{k}
$$

It is worth while to mention that for a very low damping $(\zeta \ll 1)$, the frequency of damped vibration is $f_{d n}$ nearing the natural frequency $f_{0 n}\left(f_{d n} \approx f_{0 n}\right)$. Therefore, the dimensionless damping coefficient in function of the amplitude for the window having the width of $k$ periods can be expressed by an approximate formula

$$
\left.\zeta(Z)\right|_{Z=\frac{1}{2}\left(Z_{n}+Z_{n+k}\right)} \cong \frac{1}{2 k \pi} \Delta_{n k}
$$

In the event when value of the damping coefficient is difficult to estimate, an exact formula will be recommendable. Recalling the relationship between the natural frequency with no damping $f_{0 n}$ and with damping $f_{d n}$, the dimensionless damping factor is derived in function of the amplitude for the window having the width equal to $k$ periods

$$
\left.\zeta(Z)\right|_{Z=\frac{1}{2}\left(Z_{n}+Z_{n+k}\right)}=\frac{\Delta_{n k}}{\sqrt{\Delta_{n k}^{2}+4 k^{2} \pi^{2}}}
$$

Accordingly, the natural frequency $f_{0}$ can be written by the formula

$$
\left.f_{0}(Z)\right|_{Z=\frac{1}{2}\left(Z_{n}+Z_{n+1}\right)}=\frac{f_{d}(Z)}{\sqrt{1-\zeta^{2}(Z)}}
$$

Thus, we get the dimensionless damping coefficient $\zeta$ and the natural frequency $f_{0}$ in the window having the width of $k$ periods and starting at the time instant $t_{n}$ when the amplitude $Z_{n}$ occurs. This procedure marks the end of Step 3.

In Step 4, it is checked whether the number being the difference between the number $N$ of sought amplitudes and the window width $k$ should be greater than the ordinal number of the iterative procedure $i$ (for example $N-k>i$ ). When this condition is not satisfied, the indicator $i$ is incremented and the window is moved to the right with the step equal to one period of damped vibration (to the next amplitude $Z_{n+1}$ ), thus marking the beginning of the next iteration. The calculation procedure for the new window position is identical as in Step 3. The window is moved until the moment when the amplitude $Z_{n+k}$ in the window is the ultimate amplitude thus found, in other words, until the condition $N-k>i$ gets satisfied. The number of the thus determined values of $\zeta(Z)$ and $f_{0}(Z)$ is associated with the number of observed amplitudes $Z$ and width of the window $k$, and is equal to $N-k$. Having the algorithm procedure completed, the actual value of $i$ implicates the number of the thus determined values of $\zeta(Z)$ and $f_{0}(Z)$. The width of the window $k$ determines the shape of $f_{0}(Z)$ and $\zeta(Z)$ plots. The parameter $k$ operates as a low-pass filter - averaging and smoothing both the natural frequency $f_{0}$ and dimensionless damping coefficient $\zeta$ versus vibration amplitude $Z$.

Step 5: taking into account the variable window width $k$, the thus derived characteristics can be smoothed demonstrating how the natural frequency $f_{0}$ and the dimensionless damping coefficient $\zeta$ should vary in function of the amplitude $Z$. Then the parameters are established. They express the beam vibration response depending on the field strength and the actual location of magnetic field action. The thus calculated parameters are the equivalent natural frequency $f_{0 z}$ and the equivalent damping coefficient $\zeta_{z}$. Both quantities are computed as the arithmetic mean of dimensionless damping coefficients and natural frequencies computed for each window, using formulas (4.4) and (4.5). The final formulas expressing the equivalent natural frequency $f_{0 z}$ and the equivalent damping coefficient $\zeta_{z}$ are written as

$$
f_{0 z}=\frac{1}{N-k} \sum_{i=1}^{N-k} f_{0_{i}} \quad \zeta_{z}=\frac{1}{N-k} \sum_{i=1}^{N-k} \zeta_{i}
$$


Thus the final Step 5 is completed. The parameters $f_{0 z}$ and $\zeta_{z}$ characterising vibration of the beams filled with MRF1 and MRF2 are derived for all cases when the free vibration responses have been registered. The same procedure is applied to derive the parameters $f_{0 z}$ and $\zeta_{z}$ for Beam 3 and Beam 4.

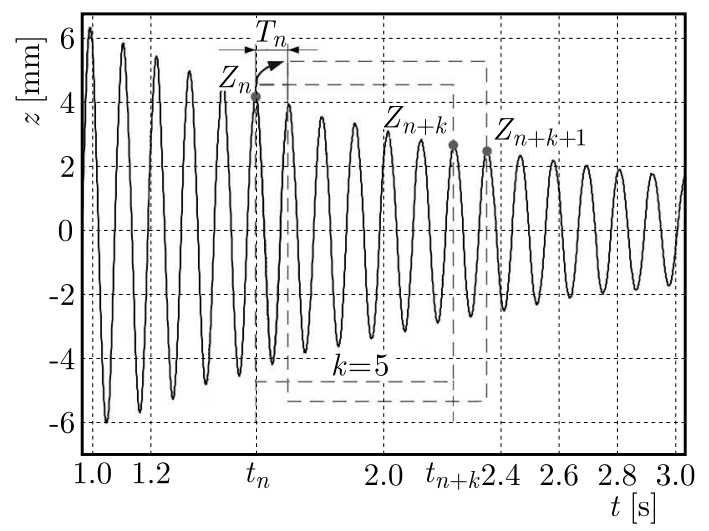

Fig. 5. Procedure of finding the natural frequency and dimensionless damping coefficient

This data processing algorithm would yield characteristics of stiffness and damping in function of the vibration amplitude $Z$ and of the equivalent dimensiomless stiffness and equivalent damping coefficient in function of the magnetic field strength and the electromagnet position.

\section{Results and discussion}

Stiffness and damping characteristics in function of the vibration amplitude $Z$ are not included in this paper. However, their determination has been necessary to calculate the equivalent fundamental frequency and the equivalent damping coefficient in function of the magnetic field strength and the electromagnet position. Recalling respective formulas (4.6), the equivalent fundamental frequency $f_{0 z}$ and the equivalent dimensionless damping coefficient $\zeta_{z}$ have been derived accordingly. The variability pattern of the equivalent fundamental natural frequency $f_{0 z}\left(H, y_{m}\right)$ and the equivalent dimensionless damping coefficient $\zeta_{z}\left(H, y_{m}\right)$ in function of the magnetic field strength $H$ and the electromagnet position $y_{m}$ for Beam 1 and Beam 2 are shown in Figs. 6 and 7. The actual value of the equivalent fundamental natural frequency $f_{0 z}$ is derived from formula $(4.6)_{1}$ whilst the equivalent dimensionless damping coefficient from formula $(4.6)_{2}$. The domain of the function $f_{0 z}\left(H, y_{m}\right)$ and $\zeta_{z}\left(H, y_{m}\right)$ becomes the variability range of the parameters $H$ and $y_{m}$. The values of parameter $H$ vary from 0 to $148 \mathrm{kA} / \mathrm{m}$, and the variability range of $y_{m}$ is from 40 to $140 \mathrm{~mm}$. This domain results from the test conditions. The characteristics $f_{0 z}\left(H, y_{m}\right)$ for Beam 1 and Beam 2 are shown in Fig. 6. In the off-state when $H=0$, the equivalent fundamental frequency $f_{0 z}\left(0, y_{m}\right)$ for Beam 1 and Beam 2 is 9.35 and $8.8 \mathrm{~Hz}$, respectively. In the off-state the iron particles suspended in the carrier fluid are able to move freely. The magnetic moments associated with each particle of the MRF are directed randomly. That is why the difference in $f_{0 z}$ for Beam 1 and Beam 2 can be attributable to the difference in the beam mass (Beam 1 is lighter than Beam 2 due to a lower iron particle content). Therefore, the equivalent natural frequency $f_{0 z}$ registered for a lighter beam-Beam 1 is higher. For fully filled beams operated in the on-state, the critical electromagnet position is observed at $y_{m}=100 \mathrm{~mm}$. In plots in Fig. 6, this boundary is indicated by a grey plane dividing the stiffness characteristics into two areas. Area 1 is contained between the electromagnet positions from 40 and $100 \mathrm{~mm}$. This separation is associated with the influence of the magnetic field strength $H$ on the value of the equivalent fundamental frequency $f_{0 z}$. When the field strength is the maximum 
$H=148 \mathrm{kA} / \mathrm{m}$ and the electromagnet is moved from the beam fastening $\left(y_{m}=40 \mathrm{~mm}\right)$ towards its free end, the frequency $f_{0 z}$ tends to increase (the stiffening effect). This increase continues until the electromagnet reaches its critical position $y_{m}=100 \mathrm{~mm}$. When the electromagnet is moved still further from the beam fastening, the frequency $f_{0 z}$ tends to decrease. For the electromagnet position $y_{m}=140 \mathrm{~mm}$ and magnetic field strength $H=148 \mathrm{kA} / \mathrm{m}$, the equivalent frequency $f_{0 z}$ is lower than in the off-state. For any electromagnet position within area 1 , an increase in the magnetic field strength results in an increase in the equivalent fundamental frequency $f_{0 z}$. Within this area, the stiffening effect is observed for the two beams. The increase of the equivalent fundamental frequency is also observed in area 2 for the magnetic field strength $H$ falling in a specific range. For Beam 1, this range is from $H=0$ to $H=75 \mathrm{kA} / \mathrm{m}$, for Beam 2 is from 0 to $45 \mathrm{kA} / \mathrm{m}$. A further increase in $H$ leads to a decrease in $f_{0 z}$ for the two fully filled beams. The beam damping effect predominates over the stiffening effect. Two hypotheses have been put forward to account for this behavior.
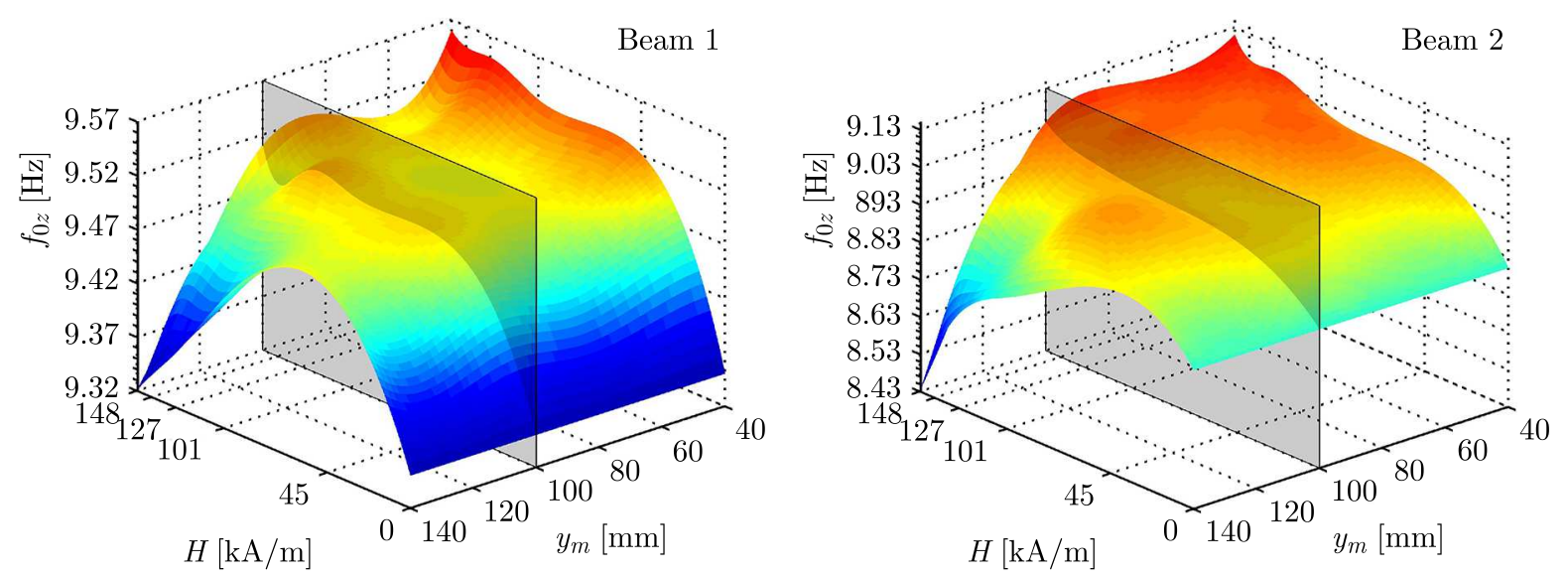

Fig. 6. Equivalent natural frequency $f_{0 z}$ vs. the magnetic field strength $H$ and electromagnet position $y_{m}$

The first hypothesis emphasises the increasing influence of field non-homogeneity on the edges of the area between the electromagnet poles. The influence of field non-homogeneity on the beam motion is enhanced with the increasing amplitude of vibration, that is for beam points further away from the fastening (increased $y_{m}$ ). Each iron particle in the MRF has two poles which can be assigned a given 'magnetic charge'. The signs of these poles are different, therefore each particle forms a magnetic dipole. When subjected to an external magnetic field, it will be directed in accordance with the field lines. When the external field is non-homogeneous, an iron particle will be attracted to the stronger field region. Moved from its equilibrium position, the beam will move in the direction in which the modulus of the magnetic field strength vector should increase. The field forces acting upon the iron particles in the MRF act opposite to elasticity forces, thus reducing the frequency of the beam natural vibration.

In accordance with the other hypothesis, the storage modulus of the MRF in this area is smaller than the loss modulus. That is why the MRF contained in the beams exhibits properties of a viscous material rather than an elastic material (Lara-Prieto et al., 2010), which leads to a relatively fast increase in the beam damping effect, illustrated by plots of $\zeta_{z}\left(H, y_{m}\right)$ in further Sections. The extreme values of $f_{0}$ are obtained for the MRF in the on-state. The highest value of $f_{0 z}$ for fully filled beams is registered for $H=148 \mathrm{kA} / \mathrm{m}, y_{m}=40 \mathrm{~mm}$, approaching $9.56 \mathrm{~Hz}$ and $9.11 \mathrm{~Hz}$ for Beam 1 and Beam 2, respectively. The smallest value of $f_{0}$ for fully filled beams is observed for $H=148 \mathrm{kA} / \mathrm{m}, y_{m}=140 \mathrm{~mm}$, reaching $9.32 \mathrm{~Hz}$ and $8.43 \mathrm{~Hz}$ for Beam 1 and Beam 2, respectively. The equivalent natural frequency $f_{0 z}$ for MRF in the off-state $(H=0)$ being taken as the reference value, the control of parameters $H$ and $y_{m}$ allows the value of $f_{0 z}$ 
to be increased or decreased. It can be increased by $2.25 \%$ and $3.25 \%$, respectively, for Beam 1 and Beam 2, or decreased by $0.3 \%$ and $4.2 \%$. These changes in the equivalent frequency $f_{0 z}$ illustrate the influence of the iron particle content. The largest changes are observed for Beam 2 filled with MRF2 because the iron particle content in this fluid is larger.

Figure 7 plots the characteristics $\zeta_{z}\left(H, y_{m}\right)$ for Beam 1 and Beam 2. It can be readily seen that both increasing the field strength $H$ and moving the electromagnet further away from the beam fastening (increasing $y_{m}$ ) lead to an increase in the equivalent dimensionless damping coefficient $\zeta_{z}$. A sharp increase in $\zeta_{z}$ is observed in area 2, alongside a rapid decrease in $f_{0 z}$ (Fig. 6), as mentioned in previous Sections. The smallest value of the dimensionless damping coefficient $\zeta_{z}$ is registered when the MRF is in the off-state, approaching 0.0088 and 0.0090 for Beam 1 and Beam 2, respectively. The value of the equivalent dimensionless damping coefficient $\zeta_{z}$ is the highest for $H=148 \mathrm{kA} / \mathrm{m}, y_{m}=140 \mathrm{~mm}$, reaching 0.0251 for Beam 1 and 0.0739 for Beam 2 . In the off-state, there are no significant changes between the dimensionless damping coefficient values for MRF treated beams differing in the iron particle content. Application of a magnetic field with the strength $H$ and the appropriate electromagnet position $y_{m}$ make the equivalent dimensionless damping coefficient for Beam 2 increase by $721 \%$. For Beam 2, the equivalent dimensionless damping coefficient rises by $185 \%$. It shows that the increasing of the iron particle content by volume from $22 \%$ in MRF1 to $32 \%$ in MRF2 results in a significant increase in the beam damping effect.
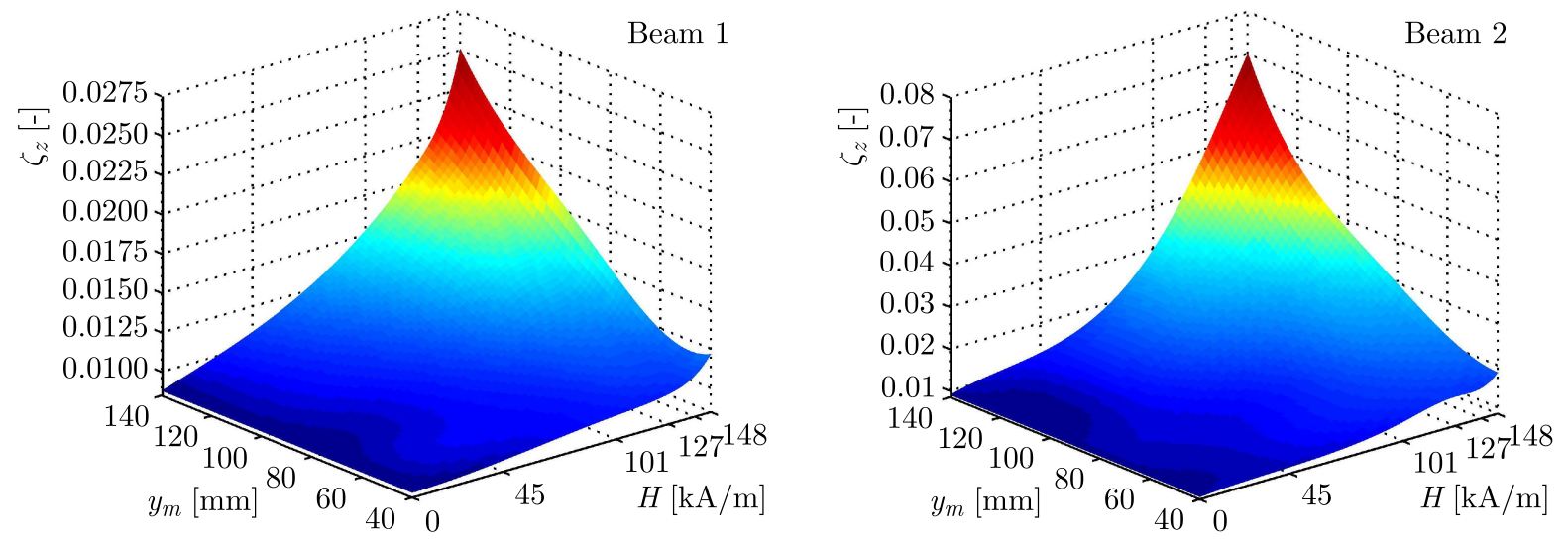

Fig. 7. Equivalent dimensionless damping coefficient $\zeta_{z}$ vs. the magnetic field strength $H$ and electromagnet position $y_{m}$

The test results provided in the present study can be compared with the results presented in the previous authors' works (Romaszko et al., 2011; Snamina et al., 2012b). There is a satisfactory convergence comparing current equivalent natural frequencies and equivalent dimensionless damping coefficients with the previous ones. The former measurements were made when the beams were not fixed to the vibration shaker armature and the magnetic field was produced by a newly engineered electromagnet. It implied satisfactory repetition, no disturbance from the shaker armature suspension, no aging, no liquid sedimentation, no leakage and recreation the approprite magnetic field strength.

Figure 8 plots the equivalent natural frequency $f_{0 z}$ and the equivalent dimensionless damping coefficient $\zeta_{z}$ for partially filled beams. Results obtained for Beam 2 for the electromagnet position $y_{m}=40 \mathrm{~mm}$ and $y_{m}=140 \mathrm{~mm}$ are also given, for easy comparison with the fully treated beams. As the iron particle content by volume for Beam 2 is higher than for Beam 3 and Beam 4 , Beam 2 exhibits lower stiffness. Increasing the magnetic field strength leads to an increase in the natural frequency (stiffening effect). Shifting the field interaction zone towards the beam free end causes the beam stiffness to decrease with an increased magnetic field strength. That is clearly seen for Beam 4 (partially filled) and Beam 2 (fully filled) when $y_{m}=140 \mathrm{~mm}$. The most 
considerable changes in the natural frequency are observed for Beam 4. For the maximum field strength $H=148 \mathrm{kA} / \mathrm{m}$, the frequency $f_{0 z}$ is reduced by $6.4 \%$ in relation to $H=0$. Experiments showed that the equivalent dimensionless damping coefficient for $H=0$ for Beam 3 was $\zeta_{z}=$ 0.0305 and for Beam $4 \zeta_{z}=0.0216$. It appears, therefore, that placing the MRF in pocket 3 in the off-state vastly reduced the potential of energy dissipation of the vibrating beam, unlike in the case when the beam was subjected to the magnetic field of strength $H>75 \mathrm{kA} / \mathrm{m}$. For the maximum field strength $H=148 \mathrm{kA} / \mathrm{m}$, the damping coefficient for Beam 4 is nearly twice as large as for Beam 3. Comparison of the equivalent dimensionless damping coefficient values $\zeta_{z}(0)$ and $\zeta_{z}(148)$ implicates that the slightest increase is registered for beams whose sections subjected to the action of magnetic field are located near the fastening point $\left(y_{m}=40 \mathrm{~mm}\right)$. For Beam 2, there is a two-fold increase of the damping coefficient when $y_{m}=40 \mathrm{~mm}$, for Beam 3 this increase is 1.5 -fold. When the beam zone affected by the magnetic field shifts towards the beam free end, the dimensionless damping coefficient increases considerably. For Beam 2, a nearly 8-fold increase is observed when $y_{m}=140 \mathrm{~mm}$, for Beam 4, nearly 5 fold. Plots shown in Fig. 9 lead us to the conclusion that it is not the volume of the MRF used that determines the actual shape of the stiffness and damping characteristics. Engineering a specially designed beam with five pockets and filling the appropriate pocket with a MRF result in major changes in the relevant characteristics.
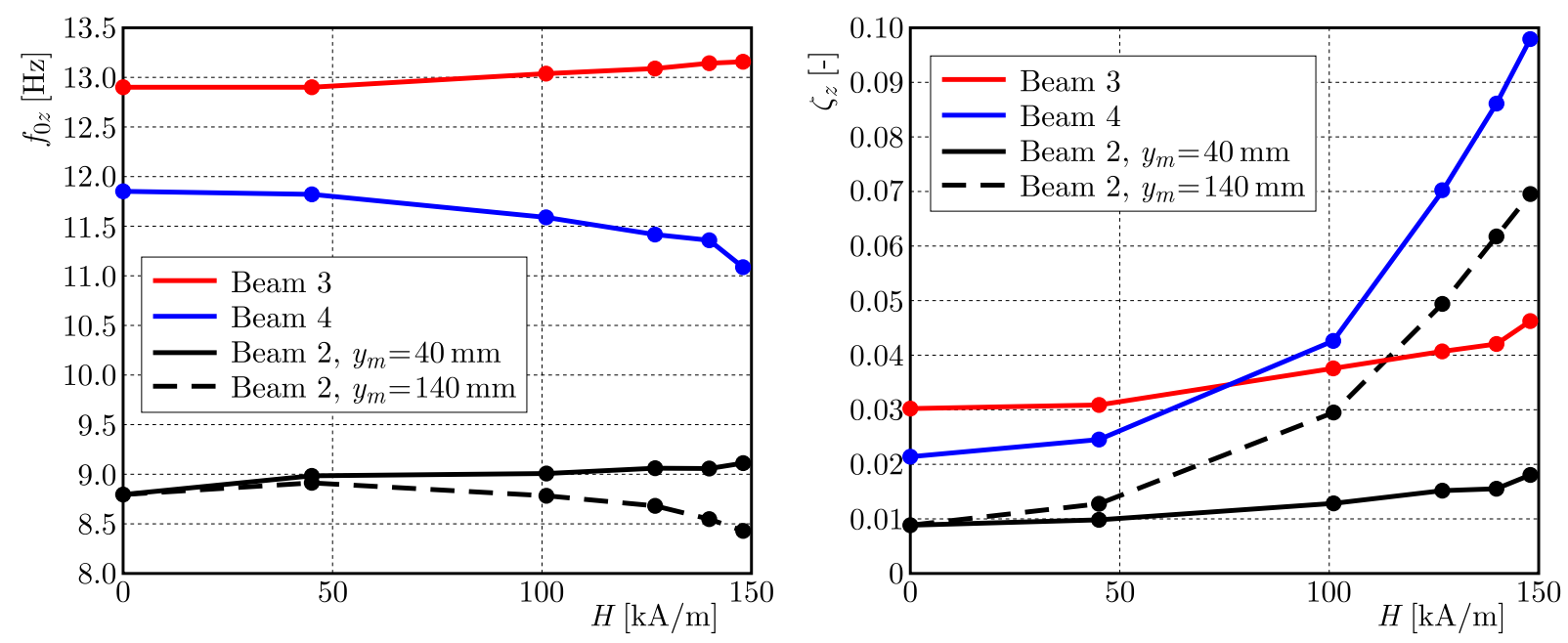

Fig. 8. Equivalent natural frequency $f_{0 z}$ and equivalent dimensionless damping coefficient $\zeta_{z}$ vs. magnetic field strength $H$

\section{Conclusions}

The study investigates the dynamic behaviour of cantilever sandwich beams (with one or five pockets) filled with MRFs varying in their iron particles content by volume. The purpose of the study is to determine stiffness and damping characteristics of the beams by measuring the free vibration response of the beams subjected to action of a magnetic field of varied strength and varied electromagnet positions. A measurement data processing algorithm has been proposed, allowing us to establish how the vibration amplitude should influence the natural frequency and the equivalent dimensionless damping coefficient. The equivalent natural frequency and the equivalent dimensionless damping coefficient have been derived accordingly.

The results of investigations lead us to the following conclusions.

- The most complex energy dissipation mechanism in beams is explained by recalling the viscous damping model and the developed data processing algorithm. 
- The natural frequency and the dimensionless damping coefficient tend to increase as the free vibrations decay. The higher the magnetic field strength and the further the electromagnet from the beam fastening point, the more significant this increase is.

- The effect of field non-homogeneity is revealed particularly when the electromagnet is in the position defined as $y_{m}>100 \mathrm{~mm}$ (see the grey-coloured plane in the stiffness and damping plots). This non-homogeneity causes a rapid increase in the dimensionless damping coefficient. At the same time, the natural frequencies of Beam 1, Beam 2, Beam 4 decrease.

- Plots of the natural frequency and the equivalent dimensionless damping coefficient clearly show that for the beam containing the MRF with a higher solids content, the variability range of the natural frequency and of the dimensionless damping factor is wider. It appears that the actual shape of the stiffness and damping characteristics for Beam 1, Beam 2 (fully filled with the MRF) and Beam 3, Beam 4 (partially treated) is associated with the location of the MRF in the beam (pocket number), not with the MRF volume.

- In the case of partially filled beams (Beam 3 and Beam 4), the factor affecting the stiffness and damping plots in the largest extent is the number (location) of MRF-containing pocket. The widest variability range of the equivalent natural frequency and the equivalent dimensionless damping coefficient is registered for Beam 4 (which has the mid pocket filled).

- The presented results are consistent with the previous ones and reveal satisfactory repetition, no disturbance from the shaker armature suspension, no aging, no liquid sedimentation, no leakage. The newly designed electromagnet enables one to recreate the appropriate magnetic field strength level.

In further work, the authors are going to investigate complex vibration modes of the beams.

Acknowledgement

This work has been supported by AGH University of Science and Technology under research program No. 11.11.130.766.

\section{References}

1. DiTaranto R.A., 1965, Theory of vibratory bending for elastic and viscoelastic layered finitelength beams, Journal of Applied Mechanics, 87, 881-886

2. Lara-Prieto V., Parkin R., Jackson M., Silberschmidt V., Kesy Z., 2010b, Vibration characteristics of MR cantilever sandwich beams: experimental study, Journal of Smart Materials and Structures, 19, 1

3. Rajamohan V., Rakheja S., Sedaghati R., 2010a, Vibration analysis of a partially treated multi-layer beam with magnetorheological fluid, Journal of Sound and Vibration, 329, 3451-3469

4. Rajamohan V., Ramamoorthy M., 2012, Dynamic characterization of non-homogeneous magnetorheological fluids based multi-layer beam, Applied Mechanics and Materials, 110-116, 105-112

5. Rajamohan V., Sedaghati R., Rakheja S., 2010b, Optimum design of a multilayer beam partially treated with magnetorheological fluid, Journal of Smart Materials and Structures, 19, 1

6. Rajamohan V., Sedaghati R., Rakheja S., 2010c, Vibration analysis of a multi-layer beam containing magnetorheological fluid, Journal of Smart Materials and Structures, 19, 1

7. Rajamohan V., Sedaghati R., Rakheja S., 2011, Optimal vibration control of beams with total and partial MR-fluid treatments, Journal of Smart Materials and Structures, 20, 11 
8. Romaszko M., 2013, Free vibration control of a cantilever MR fluid based sandwich beam, Proceedings of the 14th International Carpathian Control Conference (ICCC), Rytro, Poland, May 26-29, 2013

9. Romaszko M., LACNy L., 2015, Magnetic field analysis of the actuator in a semi-active vibration control of the beam with MR fluid, Applied Mechanics and Materials, 759, 37-44

10. Romaszko M., Pakula S., Sapinski B., Snamina J., 2011, Vibration parameters of sandwich beams with two types of MR fluid, Mechanics and Control, 30, 3, 151-156

11. Romaszko M., Sapinski B., Sioma A., 2015, Forced vibrations analysis of a cantilever beam using the vision method, Journal of Theoretical and Applied Mechanics, 53, 1, 243-254

12. Romaszko M., Snamina J., Pakula S., 2014, Composite beam's parameters identification based on frequency responses, Proceedings of the 15th International Carpathian Control Conference (ICCC), Velke Karlovice, Czech Republic, May 28-30

13. Snamina J., Sapinski B., WszoŁek W., Romaszko M., 2012a, Investigation on vibrations of a cantilever beam with magnetorheological fluid by using the acoustical signal, Acta Physica Polonica A, 121, 1-A, 188-190

14. Snamina J., Sapinski B., Romaszko M., 2012b, Vibration parameter analysis of sandwich cantilever beams with multiple MR fluid segments, Engineering Modeling, 12, 43, 247-254

15. Sun Q., Zhou J.X., Zhang L., 2003, An adaptive beam model and dynamic characteristics of magnetorheological materials, Journal of Sound and Vibration, 261, 465-481

16. Yalcintas M., DAi H., 1999, Magnetorheological and electrorheological materials in adaptive structures and their performance comparison, Journal of Smart Materials ans Structures, 8, 560-573

17. Yalcintas M., Dai H., 2004, Vibration suppression capabilities of magnetorheological materials based adaptive structures, Journal of Smart Materials and Structures, 13, 1-11

18. YeH Z.F., SHIH Y.S., 2006, Dynamic characteristics and dynamic instability of magnetorheological material-based adaptive beams, Journal of Composite Materials, 40, 1333-1359 